\title{
Los sentimientos morales de la «tristeza» china: Una ilustración del acercamiento del Metalenguaje Semántico Natural (MSN) al análisis de algunas emociones chinas «básicas»
}

\author{
ZHENGDAO YE * \\ Universidad Nacional de Australia \\ «Los hombres son cercanos entre sí por naturaleza. Divergen \\ como resultado de prácticas repetidas»
}

Confucio (Analextas, XVII:2)

RESUMEN. Este estudio emprende, desde el marco del Metalenguaje Semántico Natural (MSN) desarrollado por Wierzbicka y algunos colegas, un detallado análisis semántico contrastivo y comparativo de dos conceptos de emoción: bei y ai (a menudo glosados de manera intercambiable como tristeza, pena y dolor), que son considerados como emociones básicas en los textos filosóficos chinos tradicionales. En él se demuestra que: $a$ ) de ninguna manera son intercambiables, ni equivalentes a la idea occidental de tristeza, $b$ ) son artefactos de la cultura china, conformados por las experiencias morales y sociales del pueblo chino y su visión de la vida y del universo. En esencia, bei comporta una visión fatalista y $a i$ es una emoción moral. Esto lleva a la autora a poner en duda el postulado de universalidad basado en la visión arraigada en la lengua inglesa, y llama a los investigadores a prestar atención al marco analítico empleado en la búsqueda de aspectos universales de las emociones humanas.
Abstract. This study undertakes, under the framework of Natural Semantic Metlanguage (NSM) developed by Anna Wierzbicka and colleagues, a detailed contrastive and comparative semantic analysis of a pair of emotions concepts: bei and ai (often glossed interchangeably as sadness, sorrow, and grief), which are considered to be basic emotions in traditional Chinese philosophical texts. It illustrates that (a) they are by no means interchangeable, nor are they equivalent of the Western idea of sadness, (b) they are artifacts of the Chinese culture, shaped by Chinese people's social and moral experiences, and their view of life and the universe. Essentially, bei encompasses a fatalistic view, and $a i$ is a moral emotion. This leads the author to challenge the claims of universality based on the view entrenched in the English language, and urges researchers to pay attention to the analytical framework employed in the search for universal aspects of human emotions.

* Traducción de Silvia Kiczkovsky Yankelevich. 


\section{INTRODUCCIÓN}

Las últimas dos décadas han sido testigos de un renovado interés por el estudio de las emociones a través de varias disciplinas (cf., e. g., Shveder and Levine, 1984; White and Kilpatrick, 1995; Lewis and Haviland, 1993; Ekman and Davidson, 1994; Marks and Ames, 1995). Esta ola de interés por las emociones ha abierto también una nueva fase en la búsqueda de la «unidad psíquica del ser humano» - en el contexto actual del estudio de las emociones, la universalidad de los sentimientos humanos-. En particular, han aparecido numerosos psicólogos con diferentes listas de emociones básicas que se consideran universales. Estas listas están compuestas por «emociones básicas» en un rango que va de cinco a ocho emociones y que incluye, por ejemplo, alegría, tristeza, miedo, enojo, aversión y vergüenza (cf. Van Brakel, 1993, y Plutchik, 1994, para las diferentes listas de emociones básicas propuestas). Estas listas son tratadas como si fueran un legítimo punto de partida teórico desde el cual se pudieran describir las emociones humanas como diferentes. Podríamos preguntarnos si estas «emociones básicas» propuestas, que están basadas en la lengua inglesa, podrían extenderse a otras lenguas y a otras culturas. ¿Cómo sería el cuadro de las «emociones básicas» si fuera expresado en otras lenguas? ¿Cómo hacen los investigadores para comparar conceptos entre lenguas y culturas sin imponer sus propios valores culturales? Éstas son cuestiones importantes y urgentes que deben ser consignadas si se quiere determinar los universales de los sentimientos humanos.

Con estas cuestiones en mente este trabajo se plantea llevar a cabo un análisis semántico exhaustivo de dos conceptos de emoción que son considerados «básicos» en los textos filosóficos y morales tradicionales chinos bei y $a i$, a menudo tradu- cidos de manera intercambiable como tristeza, pena y dolor ${ }^{1}$. Al comparar y contrastar estos conceptos chinos de emoción con sus supuestas contrapartes en inglés y entre ellos, se llega a ilustrar, desde el punto de vista de la lengua china, que los investigadores deben estar alertas a las cuestiones de lenguaje, con frecuencia descuidadas pero muy significativas, en su búsqueda de los aspectos universales de las emociones humanas, y prestar particular atención al lenguaje que utilizan al postular universales. Antes de continuar para observar de cerca el significado de bei y $a i$, voy a introducir la herramienta metodológica que se emplea a lo largo de todo este trabajo al definir y analizar los conceptos de emoción -Metalenguaje Semántico Natural (MSN) - y voy a ubicarlo en el contexto de las discusiones actuales sobre los problemas de la traducción en el estudio de las emociones.

\section{LOS PROBLEMAS \\ DE TRADUCCIÓN EN EL ESTUDIO DE LA EMOCIÓN}

Entre los muchos problemas metodológicos que conciernen al estudio del léxico de las emociones y al estudio del lenguaje de las emociones en general [cf., Ye 2000a, en prensa, $a$ ), $b$ )], la posibilidad y la validez de la traducción sigue siendo el central. Para describir el significado de un concepto, los investigadores se enfrentan a menudo con un dilema. Si las traducciones son utilizadas para explicar un concepto perteneciente a otra cultura, generalmente no le hacen justicia a su significado original. Un concepto es casi siempre específico a una cultura e intraducible (cf., Rosaldo, 1980; Lutz, 1985, 1987, 1988, 1995; Wierzbicka, 1986, 1992, 1999; Russel, 1991; Schwartz, White, and Lutz, 1992; Goddard, 1997; Athanasiadou y Tabakowska, 1998). Si se utiliza un con- 
cepto autóctono, surge la cuestión de cómo estos conceptos aparentemente específicos a una cultura e indefinibles pueden ser transmitidos a aquellos que no pertenecen a esa cultura. Lo que se necesita es una herramienta descriptiva e interpretativa, un metalenguaje, que les permita a los investigadores explicar el significado pleno de un concepto en cualquier cultura y al mismo tiempo hacerlo desde una posición culturalmente independiente. Además, el metalenguaje debe ser culturalmente independiente, de modo tal que provea un trasfondo común para otras comparaciones. Esta herramienta metodológica de la lingüística es el marco analítico del Metalenguaje Semántico Natural (MSN) que se basa en un conjunto de universales léxicos intuitivamente comprensibles y autoexplicables. La aplicación del MSN al análisis de los conceptos de emoción chinos ilustra cómo este método lingüístico nos permite allanar estos problemas.

\section{EL METALENGUAJE SEMÁNTICO NATURAL}

El Metalenguaje Semántico Natural (MSN) ha sido desarrollado por Anna Wierzbicka y sus colegas a lo largo de tres décadas de investigaciones empíricas en diferentes lenguas (cf., Goddard, 1998;
Wierzbicka, 1972, 1980, 1996; Goddard y Wierzbicka, 1994, en prensa). El MSN incluye sesenta primitivos semánticos universalmente aplicables y empíricamente comprobados («el alfabeto de los pensamientos humanos») tales como DECIR, PENSAR, SENTIR, SABER, QUERER, YO, TÚ, BUENO, MALO, HACER y SUCEDER. Estos conceptos universales humanos, en palabras de Wierzbicka (1995:20), toman «forma léxica en todos los lenguajes del mundo... Pueden funcionar como bloques elementales, en términos de los cuales, todos los otros conceptos, sin importar lo idiosincráticos y únicos que sean, pueden ser definidos», y hay una «mini-gramática que especifica las reglas de su combinación». En tanto este metalenguaje está hecho de lenguaje natural y toma forma en universales léxicos, en lugar de estar basado en palabras técnicas o símbolos, puede ser intuitivamente comprendido y rápidamente traducido a otras lenguas. En cierto sentido, en cada lenguaje existe un «mini-lenguaje», en términos del cual los significados complejos dentro de ese lenguaje pueden ser explicados, y estos mini-lenguajes se corresponden tanto en su léxico como en su gramática. La realización de los exponentes del MSN en inglés y en español es presentada en el siguiente cuadro (Travis, en prensa):

\author{
Sustantivos \\ I: YO. \\ YOU: USTED. \\ SOMEONE: ALGUIEN. \\ PEOPLE: GENTE. \\ SOMETHING: ALGO. \\ Predicados mentales \\ THINK: PENSAR; \\ KNOW: SABER. \\ WANT: QUERER. \\ FEEL: SENTIR. \\ SEE: VER. \\ HEAR: OÍR.
}

Primitivos Semánticos Propuestos (inglés y español) ${ }^{2}$

Habla

SAY: DECIR.

WORD: PALABRA.

Acciones, eventos, movimientos

DO: HACER.

HAPPEN: PASAR.

MOVE: MOVERSE.

Existencia y vida

THERE IS: HAY.

HAVE: TENER.

LIVE: VIVIR.

DIE: MORIR. 
Determinadores y cuantificadores

THIS: ESTO.

THE SAME: LO MISMO.

OTHER: OTRO.

ONE: UNO.

TWO: DOS.

ALL: TODO.

SOME: ALGUNOS.

MANY/MUCH: MUCHO.

Tiempo

WHEN/TIME: CUÁNDO/TIEMPO.

NOW: AHORA

BEFORE: ANTES DE.

AFTER: DESPUÉS DE.

A LONG TIME: MUCHO TIEMPO.

A SHORT TIME: POCO TIEMPO.

FOR SOME TIME: POR UN TIEMPO.

MOMENT: MOMENTO.

Espacio

WHERE/PLACE: DÓNDE/SITIO.

HERE: AQUÍ.

ABOVE: ARRIBA DE.

BELOW: DEBAJO DE

\section{EVIDENCIA LINGÜÍSTICA}

El análisis semántico estará basado en la evidencia lingüística tomada de recursos literarios populares. Ésta incluye ejemplos textuales conocidos, ítems léxicos convencionalizados, frases y expresiones idiomáticas (en particular chengyu generalmente expresiones idiomáticas de cuatro letras) que son familiares a los oídos chinos y que sirven como entradas léxicas en diccionarios tan autorizados como Xiandai Hanyu Cidian (XHC). Tal como fue señalado por Qian (1998), ningún estudio sobre las emociones puede permitirse pasar por alto las obras narrativas y poéticas porque los escritores de estas obras son muy perceptivos. A través de la perspicacia de muchos escritores, los estudiosos de la emoción pueden llegar a tener un acceso directo al lenguaje de las emociones, a las expresiones y al «mundo intencional» de los hablantes. Es poco sorprendente que el acercamiento literario haya sido siempre
INSIDE: DENTRO DE.

ON (ONE) SIDE: A (UN) LADO.

NEAR: CERCA DE.

FAR: LEJOS DE.

Conceptos lógicos

BECAUSE: PORQUE.

IF: SI.

NOT: NO.

MAYBE: TAL VEZ

CAN: PODER.

Existencia y vida

GOOD: BUENO.

BAD: MALO.

BIG: GRANDE.

SMALL: PEQUEÑO.

Intensificador y aumentativo

VERY: MUY.

MORE: MÁS.

Taxonomía, partonimia, similitud

PART (OF): PARTE (DE).

KIND OF: TIPO DE.

LIKE: COMO.

usado como una herramienta importante por los investigadores para lograr una comprensión empírica del universo emocional de los seres humanos. Por ejemplo, en su Anthropologie in Pragmatischen Hinsicht, Kant basó su discusión sobre las emociones en materiales literarios del drama y la ficción [Kant, 1974/(1798)]. El psicólogo Klineberg (1938) estudió las emociones chinas examinando literatura china, en particular Hongloumeng ( $U n$ sueño de mansiones rojas»), la más popular e importante novela en la historia de la literatura china ${ }^{3}$. Los recursos literarios proveen ejemplos concretos y contextos, e ilustran la esfera de uso de los términos que expresan las emociones que estamos discutiendo. En el contexto chino, donde la literatura juega un importante papel logrando la continuidad y homogeneidad de la cultura y la sociedad, y siempre ha sido la mejor manera de dar forma a la experiencia de vida y al espíritu cultural (cf., Creel, 1954; Chen, 1977; Quian, 1988), es indispensable examinar 
los recursos mediante los cuales se describen y expresan las emociones ${ }^{4}$. Las pruebas lingüísticas, especialmente las pruebas de colocación, serán también usadas en la discusión para justificar la postulación de los significados.

\section{LA SEMÁNTICA}

\section{DE LOS TÉRMINOS CHINOS BEI Y $A I$}

Bei y $a i$ son comúnmente traducidos como sadness, sorrow o grief (tristeza, pena o dolor), y aparecen en diferentes versiones de «emociones básicas» en textos chinos clásicos, con bei exclusivamente en textos taoístas, y ai en textos confucianos. Russel y Yik (1966) designaron a bei y a ai como sorrow (pena) al discutir cinco versiones de las «emociones básicas» del chino. Cuando se les pidió a 50 adultos chinos educados de Hong Kong que enlistaran las siete emociones básicas del proverbio ren you qiqing liuyu (lit. «la gente tiene siete emociones y seis deseos»), bei y ai fueron frecuentemente mencionadas, con una ocurrencia de 66 por 100 para ai y 14 por 100 para bei. Las traducciones al inglés de Russel y Yik fueron como sigue: $a$ — «sorrow/grief», y bei- «sorrow/sadness». Esto parece sugerir que ai es percibido por los chinos como una de las emociones más básicas; y estos dos términos chinos comparten la misma conceptualización entre ellos y también con el término inglés sadness (tristeza). ¿Será éste el caso? Comenzaremos la discusión con bei.

\subsection{Bei («una tristeza trágica/fatalista»)}

(1) Qui otoño

Du

solo

Kong

vacío

Yu

lluvia

deng

lámpara

Bai

blanco

huang

amarillo

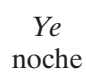

zuo

sentado

tang

vestíbulo

zhong

en
xia

xia

sobre

fa

cabello
Du

solo

bei

bei

$y u$

(futuro)

shan

montaña

cao

césped

zhong

después de todo cambio dolorost

bu ke cheng.

Sorrow over these two locks of hair, I sit alone-

In the Empty hall, almost the second watch

In the rain a fruit of the mountain falls,

Insects sing in the grasses beneath the lamp

This white hair will never change its color,

The golden elixir cannot be made...

(De Wang Wei: «Sitting alone on an Automn Night», tr. Owen, 1985:134)

Pesar sobre estas dos guedejas de cabellos, estoy sentado solo-

En el vestíbulo vacío, casi la segunda vigilia

En la lluvia una fruta cae de la montaña,

Los insectos cantan en las hierbas debajo de la lámpara

Este cabello blanco nunca cambiará su color,

El elixir dorado no puede lograrse... 
Las penetrantes y sugestivas imágenes utilizadas en este poema, como «sentado solo en una noche de otoño», «el golpe sordo de las frutas», «el lamento de los insectos» y «descubrir el primer mechón de cabello blanco», evocan un sentido de desolación, y alude al ocaso del círculo de la vida. Solo en la noche, sentado en un vestíbulo vacío, el poeta Wang Wei (701-761 d. C.), reflexiona sobre su envejecimiento que está marcado por un inevitable cambio en la vida — los cabellos negros se tornan blancos-. A medida que el vacío temporal progresa hacia la muerte de la noche (ergeng), el poeta puede percibir el ocaso de su vida. El envejecimiento, el ciclo de la vida, es inevitable e irreversible — sus cabellos blancos nunca volverán a ser negros y ningún elixir le devolverá su juventud. Debe someterse a la «ley de la naturaleza». Esta «naturalidad» del ciclo de la vida se oye a través del golpe sordo de la fruta de otoño que cae del árbol en el silencio de la montaña de la noche en la lluvia, y a través del lamento de los insectos, cuya muerte inminente se acerca con cada paso del otoño.

Cuando los cabellos negros comienzan a volverse blancos indica que una persona está entrando a shengming zhi qiu («el otoño de la vida»), y se supone que algo malo está sucediendo (a alguien). Los cabellos blancos son un signo del ocaso de la vida, y lo peor falta por llegar — la muerte-. Lo que a uno lo pone bei no es sólo algo malo que sucede en el momento, sino una incontrolable fuerza que lo dirige hacia un subsecuente suceso «trágico» determinado por la naturaleza. Además, bei está en la conciencia de quien tiene esa experiencia, quien sabe que el curso esperado de los eventos es irreversible. Nada se puede hacer para revertir el empeoramiento de los sucesos. Así, el significado de bei puede ser explicado en las siguientes líneas ${ }^{6}$ : bei

(a) X sintió algo porque $\mathrm{X}$ pensó algo

(b) a veces una persona piensa:

(c) «algo malo sucedió ahora

(d) sé que después de esto las cosas buenas no sucederán nunca más

(e) no quiero que sucedan cosas como éstas

(f) no puedo hacer nada

(g) porque sé que nadie puede hacer nada cuando suceden cosas como éstas»

(h) cuando esta persona piensa así esta persona siente algo muy malo

(i) X sintió algo así

(j) porque $\mathrm{X}$ pensó así

Los componentes (a), (b), (i) y (j) muestran un escenario cognitivo prototípico que se refiere a una situación estándar en la cual $\mathrm{X}$ siente como lo hace una persona cuando se tienen ciertos pensamientos especificables. Al enfrentar los mismos antecedentes —estímulo emocional o antecedentes situacionales-, la gente puede sentir de manera diferente porque tiene pensamientos diferentes, pero son los pensamientos prototípicos los que especifican un tipo particular de situación. Esto es consistente con las conclusiones de muchos psicólogos, que representan los conceptos cotidianos de emoción como prototipos y ven a las emociones como procesos de tipo guión con estadios identificables que involucran, como mínimo, pensamientos, sentimientos y cambios corporales (cf., Wierzbicka, 1972, 1999; Fehr y Russel, 1984; Frijda, 1986, 1995; Shaver et al., 1987).

El componente (c) («algo malo sucedió ahora») sugiere que los pensamientos de bei son una respuesta al evento reciente, cuyos efectos son todavía evidentes. El evento puede sucederle a alguien o puede haber sólo sucedido como un proceso natural, como el otoño. En cualquier caso, este evento detona una visión aún más pesimista de la situación. El componente (b) apunta a la conciencia de la continuación del deterioro de la situación 
por parte de quien tiene la experiencia: la verdadera fuente de bei. La noción de que el futuro no contiene promesas («sé que después de esto las cosas buenas no sucederán nunca más» está reflejada en los componentes tan comúnmente usados como (2), (a) y (b):

\begin{tabular}{|c|c|c|c|}
\hline (2) & a. & $\begin{array}{l}\text { bei } \\
\text { bei } \\
\text { «tragedia» }\end{array}$ & $\begin{array}{c}j u \\
\text { obra/drama }\end{array}$ \\
\hline & b. & $\begin{array}{l}b e i \\
b e i \\
\text { «pesimista» }\end{array}$ & $\begin{array}{l}\text { guan } \\
\text { visión }\end{array}$ \\
\hline
\end{tabular}

«Nunca más sucederá algo bueno», propuesto en el componente (d), indica pensamientos sobre la ausencia de un buen desenlace. Comparado con un componente como «algo malo sucederá», que refiere a la presencia de un mal resultado, el componente «no sucederá algo bueno» implica una visión pesimista más que «temerosa» del futuro.

Aunque el experienciador de bei no desea el desenlace esperado [componente (e) «no quiero que estas cosas malas sucedan»], no puede evitar que sucedan y debe aceptar el resultado irreversible. El componente (f) («no puedo hacer nada») muestra la incapacidad para revertir el curso de los eventos. Esto se debe a la comprensión fatalista de que los eventos suceden independientemente de la voluntad de la persona. El componente (g), «porque sé que nadie puede hacer nada cuando cosas como éstas suceden», refleja esta resignación. Este sentimiento de impotencia tiene alguna semejanza con pena donde «no puedo hacer nada» es también parte del significado.

La impotencia del individuo ante la «ley de la naturaleza» causa una amargura penetrante, dolor y melancolía, casi al punto del «desaliento y la crueldad», tal como se refleja en el componente «esta persona siente algo muy malo», sustentado por los siguientes componentes: bei tranquilo/frío «triste y lúgubre; sombrío; tétrico»

$\begin{array}{ll}\text { b. bei } & \text { tong } \\ \text { bei } & \text { dolor }\end{array}$

«tristeza dolorosa»

c. bei can

bei miserable/trágico/cruel «trágico»; «patético» (ZSHC: 333).

Cuando examinamos las estructuras cognitivas de tristeza y palabras relacionadas con ésta, tales como dolor y pena (véase Wierzbicka, 1999, para las definiciones de sadness, grief y sorrow), está claro que ninguno de estos términos de emoción en inglés incluye tal visión fatalista como un componente de su significado. La emoción $b e i$, entonces, está teñida por la visión china del mundo y de la naturaleza de la vida. Al definir la expresión común bei huan li he (5), Liang Shiqiu ofreció una definición iluminadora, que provee una valiosa evidencia para el significado de bei:

he
reunión

«Las penas de las partidas y las alegrías de las uniones que la vida tiene para ofrecer» (ZSHC: 333; el énfasis es mío).

Esta definición de «lo que la vida tiene para ofrecer» nos remite a los componentes (f) y (g).

Esto nos recuerda los siguientes versos expresados por Su Shi (1036-1101 a. C.), el fundador, y un representante principal, de la escuela dinámica de poesía en el género $C i$ : 


$\begin{array}{lccccc}\text { (6) } \begin{array}{l}\text { Ren } \\ \text { hombre }\end{array} & \begin{array}{c}\text { you } \\ \text { tener }\end{array} & \begin{array}{c}\text { bei } \\ \text { bei }\end{array} & \begin{array}{c}\text { huan } \\ \text { alegría }\end{array} \text { apartamiento } & \text { li } & \text { he } \\ \text { reunión } & \\ \text { yue } & \text { you } & \text { yin } & \text { qing } & \text { yuan } & \text { que, } \\ \text { luna } & \text { tener } & \text { nublado } & \text { soleado } & \text { ciclo } & \text { falta/ausencia } \\ \text { ci } & \text { shi } & \text { gu } & \text { nan } & \text { quan. } \\ \text { este } & \text { motivo } & \text { antiguo } & \text { difícil } & \text { totalidad/perfecto }\end{array}$

«El hombre se pone triste al partir; feliz al reunirse nuevamente.

La luna tiene un estado del tiempo que cambia: bueno o malo: crece y mengua

Desde los tiempos más antiguos, hasta nuestra propia cotidianeidad

La imperfección de todas las cosas — siempre ha sido así-» (ZGWG: 229).

Aun la separación y la reunión tienden a ser percibidas por el pueblo chino como sujetas a fuerzas naturales. Es obvio, a partir de estos ejemplos, que glosar bei como «pena»o «triste» no logra transmitir este importante matiz de significado que está moldeado por la característica Weltanschauung china. La expresión idiomática bei tian ming ren ilustra más claramente este concepto de emoción fatalista:

(7) $\mathrm{bei}$

$$
\begin{array}{ccc}
\text { tian } & \text { min } & \text { ren } \\
\text { cielo } & \text { piedad } & \text { hombre/gente }
\end{array}
$$

«Deplora el estado del universo y ten piedad por el destino del hombre» (HCYC: 17) (? ai tian).

Tian (cielo) tiene un estatus fatalista en la etno-teoría china de la vida (cf., Feng, 1953:34). Desde una perspectiva tradicional china, el universo está gobernado por un orden divino. Este rasgo hace a tian compatible con bei. Vinculado con tian («cielo») está ming («destino predestinado») - una omnipotente fuerza que gobierna la vida humana, de acuerdo con la creencia tradicional del pueblo chino (cf., Ye, 1998)—, que está intrísecamente vinculado con bei. Es interesante observar que tian no es compatible con ai. Cuando se pondera ming («destino predestinado») y la impotencia ante él, bei es el sentimiento más estrechamente asociado. Este punto se ilustra con el siguiente ejemplo (8):

(8) (Xiang-yung) you xiangdao ziji mingku, gang peile yige caimao shuangquan de nanren, xingqing you hao pianpiande dele yuannie zhenghou, buguo ai rizi bale. Yushi gengjia beitong, zhi kule banye. (HLM: v. 2:1487).

«Xiang-yun le recordó a ella la bondad de la anciana, después pensó en su propio mise- rable destino, ella se había casado con un hombre talentoso, bello, con una disposición jovial, pero entonces él había contraído esta fatal enfermedad que se lo llevaba día a día. Con aflicción, ella lloró la mitad de la noche» (DRM: v. 3:434)

Los ejemplos citados más arriba, principalmente el poema primero, indican que bei involucra un proceso de «transferencia» y «conmoción» momentánea [por lo tanto sólo «sentirse muy mal» en (h) sin referencia a un período de tiempo]. Quien lo experimenta, conmovido por algún estímulo externo, reacciona ante él. A este respecto, bei es muy diferente del sentimiento a largo plazo implicado por la palabra inglesa sorrow. La siguiente oración, que sirve como título para un capítulo en Hongloumeng, refleja típicamente un cambio natural que conduce intrínsecamente a bei, o al tipo de estímulo externo con el cual la emoción bei está típicamente asociada: 
$\begin{array}{llcccccc}\text { (9) } \begin{array}{l}\text { Gan } \\ \text { conmover }\end{array} & \text { qiu } & \text { shen } & f u & \text { qin } & \text { bei } & \text { wang } & \text { shi } \\ & \text { profundo } & \text { tocar } & \text { cítara } & \text { bei } & \text { pasado } & \text { cuestiones }\end{array}$

«Movido por un otoño, un citarista se lamenta por el pasado.»

La música de la cítara es desolada y pesada, como el golpe sordo de las frutas de otoño que caen sobre el piso. Representa el tono «explosivo» de bei. Es interesante observar que muchos estudiosos han mencionado que bei es un tono emocional clave en la música china (cf., Qian, 1998).

Desde los tiempos antiguos hasta ahora, la emoción de bei ha sido típicamente «sus- citada» por la estación qiu («otoño»). Beiqiu (lit. «bei otoño»), que es un ítem lexicalizado, ha sido uno de los temas más constantes e importantes en la literatura china (e.g., Xin, 1993). Song Yu, del Reino de Chu (alrededor del siglo II, a. C.), quien se cree fue la primera persona en reflejar esta visión popular de los sentimientos humanos en la literatura china, comenzó con la tradición de beiqiu:

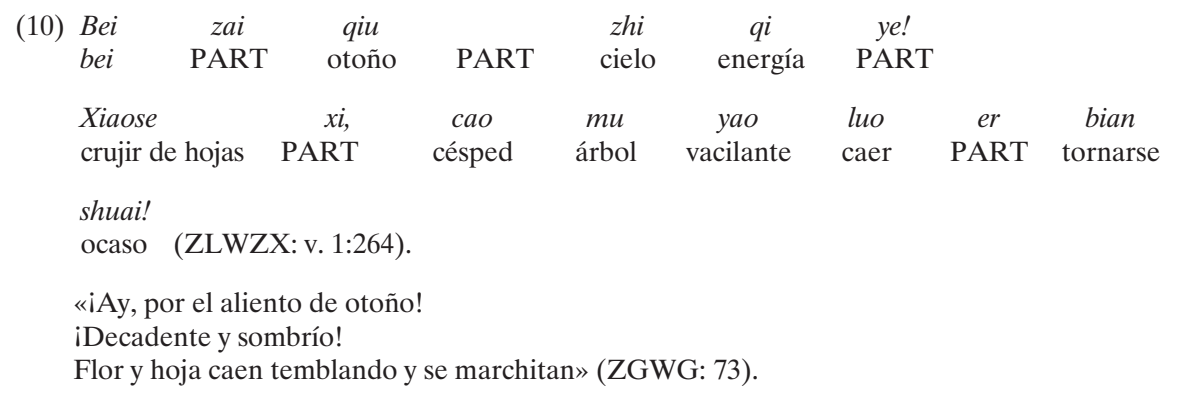

Al trazar los orígenes culturales del complejo beiqiu, Xin (1993) lo atribuye a la cosmología yin-yang característica de la visión tradicional china del universo. Esto se refleja también en la aserción de Chu Hsi (citado en Fang, 1957:56) de que «el Cielo utiliza el Yin, el Yang y los cinco elementos para transmutar todas las cosas en la forma de vida». El otoño está típicamente vinculado con la energía Yin («energía negativa»), que comienza a declinar en el proceso de la naturaleza hasta que encuentra la energía Yang («energía positiva») para generar nueva vida. Las escenas yermas y desoladas del otoño son transplantadas a un tipo de conciencia reflexiva en el individuo, que naturalmente se vuelve consciente del envejecimiento y la muerte inevitables, desde la creencia cósmica china de tian ren he yi («la unidad entre Cielo y Seres Humanos») (cf., Fang, 1957; Feng, 1953). Los procesos de generación mutua y restricción mutua en la naturaleza son independientes de la voluntad humana.

Hasta cierto punto, bei es epítome del proceso, desde que se suscita hasta la reflexión, que se construye sobre la base de la particular visión tradicional china del universo. La explicación del contenido cognitivo de bei parece sugerir una explicación plausible con respecto a por qué bei, NO $a i$, es propuesta como una de las emociones básicas en las doctrinas Taoístas, tal como el Huangdi Nei Jing («El Canon de Medicina del Emperador Amarillo», siglos V-III d. C.) y el Tao Te King, que están estrechamente asociados con la cosmología Yin-Yang. ${ }^{7}$

En beiqiu se refleja un aspecto único de las emociones chinas, a saber: el hecho de que las estaciones juegan un papel importante en las experiencias emocionales chinas. Esto se encuentra bien documentado por Liu Xie, un crítico literario 
del siglo vi, en uno de los tratados chinos más importantes sobre literatura Wen Xin Diao Long (lit. «corazón de la literatura dar forma al dragón):

Las primaveras y los otoños se suceden con la melancólica penumbra del oscuro Yin y la tranquila claridad del Yang. Y así como el semblante luminoso de las cosas físicas es cambiado en sus ciclos, también la capacidad afectiva del espíritu (hsin) es sacudida... Y cuando los cielos de otoño están altos y el aire animado adopta una claridad helada, nuestros pensamientos, sumidos en la oscuridad del Yin, tocan las cosas lejanas. El año tiene sus cosas físicas, y estas cosas tienen su rostro; nuestras emociones cambian por estas cosas, y desde las emociones llega el lenguaje. La caída de una hoja solitaria encuentra su lugar en nuestra comprensión [y sabemos que el otoño está llegando] ${ }^{8}$.

Para el pueblo chino, pensar en el otoño es pensar en sus correlativos en el ciclo de vida humano, en el ciclo dinástico, y en todos los dominios de la vida (Owen, 1985:22; Xin, 1993; Zhang, 1989). En la conciencia cultural china, el tiempo, los mayores cambios (estacionales) en la naturaleza y el movimiento de la historia son vistos como unificados, operando con un patrón cíclico. Claramente, la contraparte más cercana a «tristeza» que encontramos en el universo de las emociones chinas contiene un elemento de la visión china del universo.

\subsection{Ai («dolor/duelo éticos y altruistas»)}

Tanto bei como ai se consideran emociones cardinales en los textos tradicionales chi- nos. Sin embargo, son muy diferentes en sus estructuras cognitivas prototípicas. $A i$ está estrechamente asociado con la muerte de alguien, como se refleja en un grupo de frases relacionadas con la muerte (11). Esto puede considerarse como la primera diferencia importante entre bei y ai.

$$
\begin{aligned}
& \begin{array}{ccc}
\text { (11) a. } & a i & y u e \\
& a i & \text { música }
\end{array} \\
& \text { «música fúnebre» } \\
& \text { b. } a i \quad g e \\
& \text { ai canción } \\
& \text { «una elegía, un canto fúnebre» } \\
& \text { c. } a i \quad d a o \\
& \text { ai dolor } \\
& \text { «hacer duelo por el fallecido» } \\
& \text { d. mo silencioso } a i \\
& \text { «permanecer en tributo de silencio } \\
& \text { (por la muerte de alguien)» } \\
& \text { e. } a i \quad z i \\
& \text { ai hijo } \\
& \text { «un varón privado de su padre» } \\
& \text { f. } a i \quad s i \\
& \text { ai pensamiento } \\
& \text { «recuerdos tristes (del difunto)» }
\end{aligned}
$$

Es interesante observar que, en Hongloumeng, uno de los clásicos más populares chinos (que he examinado en detalle en Ye, 2000a), ai ocurre sólo en situaciones relacionadas con la muerte. Por ejemplo, cuando su madre muere, se dice que el personaje Dai-yu está shou sang jin ai (12a), y ai tong guo shang (12b):
(12)
$a i$
$\begin{array}{ccccc}\text { b. } & a i & \text { tong } & \text { guo } & \text { shang } \\ & a i & \text { amargo } & \text { exceder } & \text { herida }\end{array}$

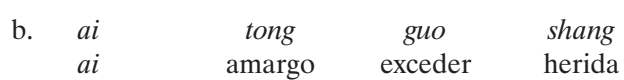 «El dolor produjo una modificación en la salud.»


El vínculo cercano entre ai y muerte parece sugerir dolor, que está prototípicamente conectado con muerte. Sin embargo, frases como ai si (lit. «ai pensamientos», «recuerdos tristes del difunto») y ai ge (lit. «ai canción», «una elegía») sugiere fuertemente que los pensamientos de $a i$ están focalizados no sobre el evento de pérdida, sino sobre la otra persona. Así, ai es muy diferente de dolor. Los pensamientos prolongados, profundos y de compasión hacia la persona fallecida son elementos claves en el significado de $a i$.

Aunque ai está prototípicamente vincu-

(13) $A i$ misheng zhi duo ai pueblo parte mucho

«Mucho he suspirado y enjugado mis lágrimas por ver a mi pueblo doblegado ante las aflicciones y los miedos» (ZGWG:67).

Lo que aflige a Qu Yuan es la desdicha que le sobrevino a la población y su prolongado sufrimiento. Una persona exiliada puede estar triste al pensar en la desdicha

(14) $a$

$$
\begin{array}{cccc}
a i & \text { guo } & a i & \text { min } \\
a i & \text { nación } & a i & \text { pueblo } \\
\text { «afligirse } & \text { por su nación y } & \text { por su pueblo» }
\end{array}
$$

En (14), el país está personificado. Los pensamientos están muy focalizados sobre el pueblo.

Esta gama de usos para ai puede encon- lado con muerte, puede también referir a otra situación donde la persona está sufriendo como resultado de un evento desastroso. Por ejemplo, en su famoso $A i$ Jiangnan Fu ( Acerca del dolor de Jiangnan»), Yu Xin (513-581 d. C.), exiliado después del hundimiento de la dinastía, expresa su ai por la caída de la dinastía, no en términos de "pérdida» personal, sino de sufrimiento de la nación [ZLWZX: v. 2(1):224]. En el exilio y pensando en su pueblo que estaba sufriendo, Qu Yuan (siglo III) escribió los siguientes versos en su famoso poema «Li Sao»:

de la nación y el sufrimiento de su pueblo, como se refleja en la siguiente frase convencionalizada:

$$
\begin{aligned}
& \text { (15) } A i \text { wo zheng fu, du wei feimin } \\
& \text { ai mi gente reclutada solamente pasivo inhumano trato }
\end{aligned}
$$

«iAy, por nosotros soldados, tratados como si no fuéramos personas! (de $\mathrm{He}$ Cao $\mathrm{Bu}$ Huang, en ZGWG).

En (15), lo que quien lo experimenta siente como ai es la miseria de los soldados que fueron enviados en servicios de expedición y que fueron tratados muy mal.

Por un lado, la «muerte» parece ser vista como la situación prototípica cuando $a i$ está presente; por otro lado, puede ser extendido a situaciones en las que un evento desafortunado le sucede al pueblo o en que una nación (en este sentido personificada) está sufriendo. ¿Cómo está funcio- nando esto para reflejarse en el significado de $a i$ ? Utilizando el acercamiento de los prototipos y contando con el primitivo semántico COMO (LIKE), explicaré el significado de $a i$ de la manera siguiente:

$a i$

(a) $\mathrm{X}$ sintió algo porque $\mathrm{X}$ pensó algo

(b) a veces una persona piensa en otra persona de esta manera (like this):

(c) «esta persona murió 
(d) esto es muy malo para esta persona

(e) no quiero que cosas como éstas le sucedan a esta persona

(f) quiero hacer algo bueno por esta persona por esto

(g) quiero pensar en esta persona durante mucho tiempo»

(h) cuando esta persona piensa así (like this) esta persona siente algo muy malo durante mucho tiempo

(i) $\mathrm{X}$ sintió algo asî

(j) porque $X$ pensó asî

(k) cuando algo muy malo le sucede a otra persona

(l) una persona puede sentir algo así

(m) porque una persona puede pensar algo así

(n) la gente piensa: es bueno si una persona puede sentir algo así cuando otra persona muere

Esta explicación está construida alrededor de dos prototipos, con uno, formulado por los componentes (b)-(h), incrustado en otro, especificado por los componentes desde $(\mathrm{k})$ a $(\mathrm{m})$. El prototipo incrustado especifica un escenario cognitivo prototípico de cuando una persona muere; con el prototipo amplio se da cuenta de las situaciones en las que eventos muy desastrosos le suceden a otra persona. Los pensamientos y sentimientos del experienciador en tales situaciones pueden ser explicados por medio de referencias a aquellos que se dan en una situación prototípica cuando otra persona muere.

Los componentes (d) («esto es muy malo para esta persona») y (e) («no quiero que cosas como éstas le sucedan a esta persona») reflejan las características de orientación hacia el otro que aparecen en $a i$, donde el foco está sobre la desdicha de otra persona. No se cuenta como una pérdida para uno. El carácter personal de PARA MÍ es parte del significado de dolor (cf., Wierzbicka, 1999:68), pero está ausente de $a i$. Por este motivo, ai no sólo es usado en conexión con la gente que uno conoce, sino también se extiende a los compatriotas.

En relación con el componente (f) («quiero hacer algo bueno por esta persona»), bei y ai divergen fundamentalmente - la visión fatalista de bei contrasta con los pensamientos altruistas de $a i-$. Esto marca la segunda gran diferencia entre los dos. $A i$ abarca «afinidad», «compasión», o incluso «amor», lo que revela un deseo de que no le sucedan cosas malas a esa persona y un querer hacer algo bueno por el desafortunado. A este respecto, ai es mucho más activo que dolor: quien experimenta dolor no va más allá de insistir en la pérdida («quiero pensar en esto»; «no puedo pensar en otras cosas»), mientras que quien experimente ai no sólo recuerda activamente al otro individuo, sino que quiere hacer algo benéfico por él. Esta buena intención puede ser vista a través de los elaborados rituales de ofrecimientos de comida para el difunto en la tumba o en las «tablillas del alma» (lingtai) en la casa. El componente (g) («quiero pensar en esta persona durante mucho tiempo») muestra este aspecto altruista de $a i$. La volición en $(\mathrm{g})$ hace a $a i$ altamente compatible con lian («amar tiernamente/compasión), como en ai lian:

$$
\begin{array}{cc}
a i & \text { lian } \\
a i & \text { compasión/conmiseración }
\end{array}
$$

«apiadarse; compadecer; tener simpatía o compasión por» (ZSHC:151) (? bei lian). 
La diferencia entre ai lian y bei lian (en el caso de que bei lian se utilice) es que, en el primero, la emoción de lian surge de la comprensión de que un desastre le sucedió a otro, mientras que en el último, surge de comprender la impotencia de los afectados y de la inevitabilidad del evento.

Cuando una persona muere, tal vez el buen deseo de hacer cosas buenas por esta persona se cumple mejor mediante el recuerdo - «quiero pensar en esta persona por mucho tiempo» [componente $(\mathrm{h})]$ Las palabras como ai si (lit. «ai pensamientos», «recuerdos tristes del difunto») indican claramente esto. $A i$ si es un pensamiento prolongado, que subsiste y que siempre ocupa la mente de las personas ${ }^{9}$. La gente puede conmemorar a una per- sona por medios como canciones y elegías. El componente (h) no tiene ninguna sugerencia de resignación, que está presente en bei. Su «simpatía» es percibida a través de los pensamientos activos sobre la otra persona.

El aspecto ético de $a i$, que es totalmente altruista, parece emitir alguna luz sobre la propuesta de ai (no bei) como una de las emociones básicas en los textos confucianos, tales como Li Ji («El libro de los ritos») y Zuo Zhuan («Un comentario sobre Zuo») (siglo viII-v a. C.). $A i$ es valorado por Confucio y sus discípulos. Los ejemplos siguientes muestran cuando ai fue mencionado en el Lun $Y u$ de Confucio («Las Analectas»).

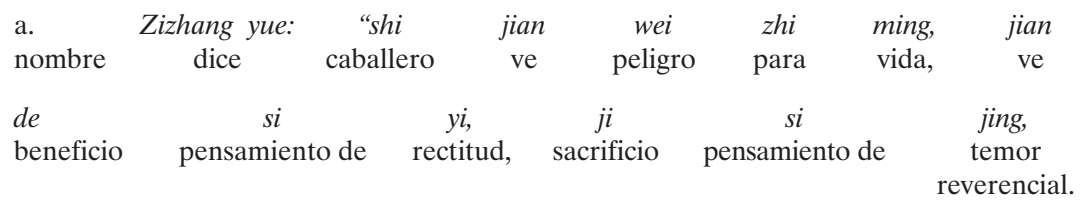

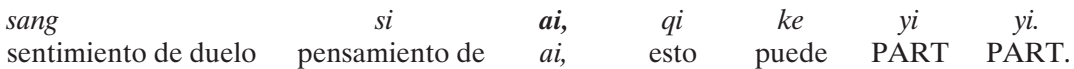

«Tsu-chang (el discípulo de Confucio) dijo: "Se puede, tal vez, estar satisfecho con un Caballero que está dispuesto a entregar su vida frente al peligro, que no olvida lo que es correcto ante la vista del beneficio, y que no olvida la reverencia durante un sacrificio ni la pena en tiempos de duelo"» (LY: 19:1)

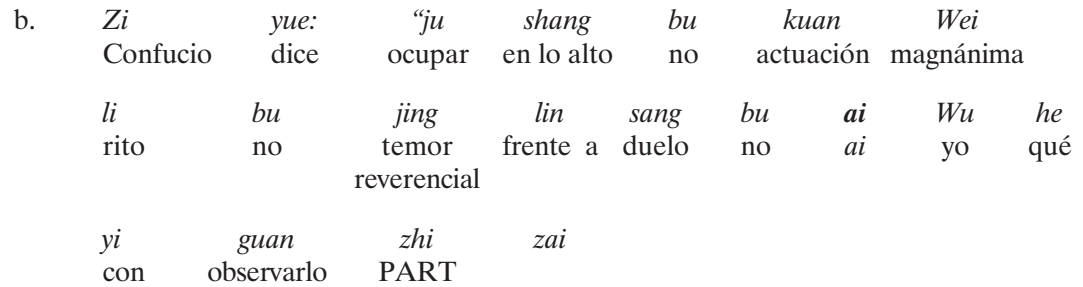

«El Maestro dijo: "¿Qué puedo observar de peor en un hombre al que le falta tolerancia cuando está en una alta posición, reverencia cuando realiza los ritos y pena cuando está de duelo?"» (LY: 3:26).

En estas citas, ai es considerado como igual a yi («rectitud») y jing («temor reverencial/respeto). En otras palabras, ai com- porta un valor moral positivo, mientras que pena o dolor no. Por lo tanto, traducir ai como «pena» o «dolor» es inapropiado y 
engañoso en estos contextos de valor específico. La idea general en estas citas es que se debe mostrar ai hacia el difunto y que cuando uno lo hace, se logra un cierto estado moral. El componente (n) en la explicación — «la gente piensa: es bueno si una persona siente algo así cuando otra persona muere»-refleja el estatuto de $a i$ como un valor moral.

La connotación moral de ai está en línea con el uso de rituales elaborados para el duelo en la cultura china. Una amplia proporción de Li Ji («El libro de los ritos») está dedicada a dar instrucciones específicas concernientes al duelo y los rituales de lamentación (cf., Chai y Chai, 1967; véase Ye, a y b para una discusión de las reglas culturales y los rituales de lamentación en chino). Las frases ai ji y ju ai reflejan una parte importante de los rituales de duelo.

$\begin{array}{cc}a i & j i \\ a i & \text { sacrificio }\end{array}$

«ritos de duelo» (ABC: 2).

b. $\quad j u$ $\begin{array}{ll}j u & a i \\ \text { levantar/apoyar } & a i\end{array}$

«lamentarse en el duelo; estar de duelo» (ABC: 332).

Una manifestación visible de $a i$ son las lamentaciones. Probablemente éste es el motivo por el cual ai se coloca a menudo con $k u$ («llanto») o $d a k u$ («gran llanto»), y por el cual bei parece estar a menudo vinculado con sonidos y voces.

Muchos antropólogos y sociólogos han observado un fenómeno inusual en la cultura china: el alto grado de uniformidad en los rituales vinculados con la muerte, como lamentaciones y duelo en toda China y a lo largo de toda su historia (e. g., Watson y Rawski, 1988). Consideran que los rituales de la muerte son algo que mantiene unida a toda la cultura china y que define la identidad del pueblo chino. Los estudiosos se sorprenden del fenómeno cultural reinante en China y quedan perplejos por las fuerzas que hay detrás de esta uniformidad y estandarización. Sugiero que no son las formas visibles que se realizan ante la muerte y su codificación lo que mantiene unificados a los chinos, sino que el nivel de valores morales más profundo asociado con la muerte de otra persona es lo que impulsa y cultiva el $l i$ externo («ritos/ceremonia/propiedad») - uno de los valores confucianos clavespara representar los sentimientos morales profundos.

La tercera diferencia entre bei y ai radica en el intenso sentimiento a largo plazo del experienciador de $a i$, y la explosiva, momentáneamente intensa conmoción, de bei. Bei erupciona como un volcán. Es más fácil para quien lo experimenta acabar con él, mientras que ai es una emoción prolongada, persistente, penetrante, que se asemeja a los tonos de la música fúnebre. También recuerda los lamentos de los pájaros moribundos en un valle con eco, o el residuo del sonido que penetra persistentemente los oídos. Ai es también acumulativo al punto de ai hui gu li:

$$
\begin{aligned}
& \text { (20) } a i \quad h u i \quad g u \quad l i \\
& a i \quad \text { destruir huesos estar }
\end{aligned}
$$$$
\text { «demacrado con dolor» (ABC: } 2) \text {. }
$$

Generalmente hablando, ai difiere de dolor en tres áreas principales: «orientación persona» vs. «orientación hacia el evento» (la pérdida); «altruista» («otra persona», «para esta persona») vs. «personal» (para mí); y prolongado («sentirse muy mal por mucho tiempo») vs. momentáneo. En este sentido, ai está más cercano en significado a duelo, en que ambos están relacionados con la muerte y que los pensamientos están centrados sobre la otra persona. Sin embargo, hay algunas diferencias importantes. En primer lugar, ai, aunque estrechamente relacionado con situaciones de muerte, no se limita exclusivamente a ellas. En segundo lugar, ai es una emoción mucho más intensa que puede causarle daño a quien la experimenta. 
En tercer lugar, la connotación moral de ai desaparece en duelo. Ai es una emoción extraordinariamente valorada en la cultura china. Su estatus como una emoción humana cardinal y la elaboración de las frases relacionadas con $a i$ son fuertes manifestaciones de su importancia en la cultura china. $A i$ ilustra los sentimientos morales y los valores del universo emocional chino.

\section{6. ¿TRISTEZA}

\section{COMO UN UNIVERSAL?}

Tristeza ha sido propuesta como una de las emociones básicas por parte de muchos psicólogos, y aparece en todas las listas de emociones humanas universales (e. g., Ekman, 1992). En este aspecto hemos visto que el análisis preciso de la estructura cognitiva de los términos de emoción chinos que se asemejan a «tristeza» y que son considerados como emociones básicas en la cultura china proveen evidencia empírica de que las emociones que el pueblo chino considera como básicas son muy diferentes a las propuestas por la lengua inglesa. Si la postulación de emociones básicas estuviera basada en la lengua china, podría incluir a bei («tristeza fatalista/trágica») y a ai («dolor/duelo ético, altruista»). Ninguno de estos términos de emoción, tal como han sido discutidos en este trabajo, tiene equivalentes exactos en inglés. No hay razón alguna para asumir que bei y $a i$ no sean tan básicos como tristeza.

Las «emociones básicas» propuestas por los chinos designan lo que los chinos consideran importante. Las emociones básicas de los chinos están conformadas por sus experiencias culturales, sociales y morales. El concepto global de bei, que resalta un sentido de impotencia ante las leyes de la naturaleza y que está coloreado por la visión cíclica de la vida y el cambio de los chinos, puede estar ausente en otras culturas. Bei es más trágico y fatalista, invo- lucra una transferencia momentánea de un estímulo externo a una reflexión interna. $\mathrm{Su}$ asociación con la naturaleza y los cambios de estación es característica de la experiencia emocional de los chinos. $A i$, aunque involucra pérdida, es ético por naturaleza, y surge de la compasión y la simpatía altruista. El inglés no comparte la misma conceptualización de la experiencia de «tristeza» china. La pretensión de que tristeza es una emoción universal está sesgada hacia la lengua inglesa. Para citar a Solomon (1995:179):

El problema, entre otros, es que tomamos la categoría de emociones como algo establecido, y después nos preguntamos si o hasta qué punto la categoría china corresponde a la nuestra. Pero, por supuesto, esto no es así, no sólo porque nosotros mismos no tenemos esta categoría establecida, sino porque no hay ninguna razón para pensar que el concepto chino - que parece tener más que ver con lo que podríamos llamar «autenticidad» que con un ámbito particular de «la mente»— tenga ninguna relación interesante con el tipo de categoría clasificatoria que pretenden tener las «emociones» en inglés.

Esto apunta a la cuestión más amplia de si las emociones universales existen. Durante siglos, los estudiosos de diferentes áreas de investigación -filósofos, psicólogos, antropólogos y lingüistas - han sido atraídos, así como atormentados, por esta búsqueda. Pero los investigadores también necesitan reconocer las limitaciones de sus métodos. Cuando la medida utilizada no es neutral, corremos el riesgo de proyectar sobre otros pseudo-universales (cf., Shwedwe, 1991, en prensa; Wierzbicka, 1986, 1999). En sus formas más extremas, asumir que los términos y conceptos del inglés son universalmente aplicables llevaría a los investigadores a descuidar los diferentes modelos de emociones de otras culturas. Lutz (1985, 1998), por ejemplo, ve el descuido de los aspectos socioculturales de emoción como una de esas consecuencias nocivas, porque los modelos occiden- 
tales del yo definen a las emociones principalmente en términos del individuo sin importar las relaciones sociales. Esto se repite en la observación de Markus y Kitayama (1994): a diferencia de Occidente, donde el yo es individualista y autónomo, en muchas sociedades orientales el yo es relacional e interdependiente. Estas diferentes orientaciones sociales y culturales resultan en diferencias observables en las emociones. Kleinman (1986) también señala que la experiencia china del yo está orientada hacia valores en contraste con el énfasis sobre la privacidad y el egocentrismo en la orientación del yo occidental. $A i$ y dolor, dos términos de emoción en dos culturas, ambos relacionados con la muerte y, sin embargo, separados por las características de orientación hacia el otro u orientación hacia sí mismo, parecen dar testimonio de la diferente orientación cultural entra las culturas china y anglo.

Rechazar completamente la búsqueda de universales de la naturaleza humana no sería una actitud constructiva. El análisis en este trabajo muestra que la búsqueda de un núcleo común entre los seres humanos no es una cuestión de todo o nada. Se puede extraer la estructura común una vez que el escenario cognitivo se explica mediante conceptos humanos universales. Por un lado, bei y ai, y tristeza tienen estructuras cognitivas muy diferentes, por el otro, comparten un núcleo semántico invariante de «algo malo sucedió/está sucediendo», que puede en ambas lenguas vincular estos términos de emoción chinos con otros conceptos de emoción que están construidos sobre esta base cognitiva. Lo básico no son los términos mismos de emoción, sino los «elementos de respuesta» comunes compartidos (Sherer, 1994:25) que pueden ser expresados en universales léxicos. Es el elemento cognitivo encontrado en las emociones el que funciona como una base para la universalidad de las emociones. La configuración de estos elementos está modelada por la cultura, y el desciframiento del significado conceptual profundo de los conceptos de emoción refleja la orientación cultural predominante.

\section{CONCLUSIONES}

En este trabajo hemos realizado un análisis semántico basado en textos de dos términos de emoción - bei y ai- que son considerados emociones básicas en China. Hemos alcanzado varias conclusiones que se pueden resumir de la manera siguiente:

1. Usando el marco de análisis semántico del MSN, se ha mostrado que estas dos emociones chinas, que se asemejan a «sadness» («tristeza») no tienen equivalentes semánticos exactos en inglés. Se pone en cuestión así la pretensión de que tristeza sea una emoción universal.

2. Enmarcar las definiciones en el MSN tiene tres ventajas principales en el estudio de las emociones. En primer lugar, hace posible la explicación de los significados desde una perspectiva internalista. En segundo lugar, las diferencias exactas y las conexiones entre conceptos, dentro de una cultura y entre culturas, pueden identificarse claramente - un punto que queda bien ilustrado al comparar, por un lado, ai y bei y, por el otro, ai y dolor-. En tercer lugar, permite que las definiciones sean traducidas a diferentes lenguas mientras se mantiene la neutralidad.

3. Los términos de emoción son como la punta de un iceberg con una gran cantidad de significado por debajo. Sacar este nivel profundo de significado a la superficie requiere no sólo de esfuerzo, sino también de una herramienta metodológica lingüística apropiada. A pesar de lo intimidante de la tarea, este estudio ha mostrado que es posible hacerlo con la ayuda del acercamiento del MSN, ya que nos permite sacar el significado a la superficie.

4. Los recursos literarios, incluyendo a los ítems léxicos, expresiones convencionalizadas y ejemplos literarios, son fuentes 
lingüísticas valiosas que no deberían pasarse por alto en la investigación de las emociones. La lexicalización de palabras, como beiqiu (lit. «bei otoño»), da testimonio de la visión de la vida china, y del papel que juegan las estaciones en la conformación de las experiencias emocionales chinas.

5. Una vez que se consigue desplegar el significado, la «lógica cultural» resulta más fácil de desentrañar. La explicación de los significados correspondientes a bei y ai aporta luz sobre la «lógica» que está detrás de sus diferentes estatus en las versiones taoísta y confuciana de las «emociones chinas básicas»-el cosmos encarnado en bei y el sentimiento moral en $a i$, y sus conexiones con la conducta social. La teoría lingüística puede contribuir al estudio de las emociones y el estudio de las emociones puede beneficiarse con una perspectiva interdisciplinaria.

\section{APÉNDICE: \\ DICCIONARIOS \\ Y REFERENCIAS LITERARIAS ORIGINALES}

ABC, Alphabetically-based computerized Chinese-English dictionary (Diccionario chino-inglés computerizado ordenado alfabéticamente), Defrancis (ed.) (1997), NSW, Allen \& Unwin.

DRM, A dream of red mansions (traducido por Hsien-yi Yang- Gladys Ynag) (1994), Beijin, Forein Languages Press (Un sueño de mansiones rojas).

HCYC, Hany chengy hanying cidian (Diccionario de expresiones idiomáticas chinas chino-inglés) (1991), Heifei, Zhongguo kexue jishu daxue chubanshe.

HLM, Hong lou meng Yue-qin Cao-E Gao, 1998/(17??), Beijin, Renmin Wenxue Chubanshe.

JYHC, Jingxuan yinghan hanying cidian (Breve diccionario inglés-chino chino-inglés) (1986), Oxford, Oxford University Press.
LY, Lun Yu-The Analects (traducido por D. C. Lau) (1983), Hong Kong, The Chinese University Press (Las Analectas). XHC, Xiandai hanyu cidian (Un diccionario de chino moderno) (1998), Beijin, Shangwu Chubanshe.

ZGWG, Zhongguo guodian wenxue gaikuang (Un compendio de literatura china clásica — con excepción de las obras maestras-), Dexin Zhang (1994), Beijin, Beijin Language and Culture University Press.

ZLWZX, Zhongguo lidao wenxue zuopin xuan (Selección de literatura china histórica), Dongrun Zhu (ed.) (1990), Shanghai, Shanghai Guji Chubanshe.

ZSHC, Zuin shuyong hanying cidian (Un nuevo diccionario práctico de palabras chino-inglés), Shiqiu Liang (1973), Taipei, The Far East Book.

\section{REFERENCIAS BIBLIOGRÁFICAS}

Athanasiadou, Angeliki, y Tabakowska, Elzbieta (eds.) (1998), Speaking of emotions: conceptualization and expression, Berlin, Mouton de Gruyter.

CaO, Xueqin, y GaO, E. (2000), Hongloumeng, Zhejiang, Zhejiang wenyi chubanshe (17 ??) (Lecturas para estudiantes de secundaria propuestos por el Ministerio de Educación Chino, Ministry of Education).

Chai, Ch'u, y Chai, Winberg (eds.) (1967), Book of Rites: an encyclopedia of ancient ceremonial usages, religious creeds, and social institutions, Nueva York, University Books.

Chappell, Hilary (1994), Mandarin semantic primitives, en GODDARD y WIERZBICKA (eds.), pp. 109-47.

- (en prensa), Universal syntax and Mandarin Chinese grammar, en GODDARD y Wierzbicka (eds.).

Chen, Lifu (1977), An introduction to Chinese culture, Taipei, The Council of Chinese Cultural Renaissance. 
Creel, H. G. (1954), Chinese thought from Confucius to Mao Tse-tung, London, Eyre \& Spottiswoode.

EkMAN, Paul (1992), «An argument for basic emotions», Cognition and Emotion, núm. 6, pp. 169-200.

Ekman, Paul, y Davidson, Richard J. (eds.) (1994), The nature of emotion: fundamentalquestions, Oxford, Oxford University Press.

FAng, Tung-mei (1957), The Chinese view of life: the philosophy of comprehensiveharmony, Hong Kong, Union Press.

Fehr, B., y Russell, James A. (1984), «Concept of emotion viewed from a prototype perspective», Journal Of Experimental Psychology: General Section, núm. 113, pp. 464-86.

Feng, Yu-lan (1953), A history of Chinese philosophy (traducción de D. Bodde), Princeton, Princeton University Press, 1934.

FriJdA, Nico H. (1986), The Emotion, New York, Cambridge University Press.

Fridda, Nico H.; SATo, Kaori, y Wiers, Reinout (1995), «Emotions and emotion words», en Russell, James A.; FERnÁNDez-Dols, José Miguel; Manstead, Antony, y Wellenkamp, J. C. (eds.), Everyday conceptions of emotion: an introduction to the psychology, anthropology, and linguistics of emotion, Dordrecht and Boston, Kluwer Academic, pp. 121-144.

GodDARD, Cliff (1997), «Contrastive semantics and cultural psychology: "surprise" in Malayand English», Culture and Psychology, núm. 3 (2), pp. 153-181.

- (1998), Semantic Analysis: A Practical Introduction, Oxford, Oxford University Press.

Goddard, Cliff, y Wierzbicka, Anna (eds.) (1994), Semantic and Lexical Universals: Theory and Empirical Findings, Amsterdam, John Benjamins.

- (en prensa), Meaning and Universal Grammar: Theory and Empirical Findings, Amsterdam, John Benjamins.
Harkins, Jean (1990), review of OrTONY, A.; Clore, G. L., y Collins, A., The Cognitive Structure of Emotions, Cambridge University Press (1988), Australian Journal of Linguistics, núm. 10, pp. 277-287.

Harkins, Jean, y WierzBicka, Anna (1997), «Language: a key issue in emotion research», Innovation in Social Sciences Research, núm. 10(4), pp. 319-31.

- (en prensa), Emotions from cross-linguistic perspective, La Haya, Mouton de Gruyder.

KANT, Immanuel (1974), Anthropology from a pragmatic point of view (traducción de Mary J. Gregor), La Haya, Nijhoff, 1799.

KlineberG, Otto (1938), «Emotional expression in Chinese literature», Journal of Abnormal and Social Psychology, núm. 31, pp. 517-520.

Kleinman, Arthur (1986), Social origins of distress and disease: depression, neurasthenia, and pain in modern china, New Haven, Yale University Press.

Lewis, Michael, y Haviland, Jeanette M. (eds.) (1993), Handbook of emotion, New York, Guiford Press.

LiAnG, Chengmou (1996), «Qiqing shuo yu xiandai qingxu xinlixue» (La teoría de las siete emociones básicas y la psicología moderna de las emociones), Nanjing Shifan Daxue Xuebao: Shehui Kexue (Journal of Nanjing Normal University: Social Sciences), núm. 4, pp. 64-7.

LuTZ, Catherine (1985), Ethnopsychology compared to what? Explaining behavior and consciousness among the Ifaluk, en White, Geoffrey M., y KiRKPATRICK, John (eds.), pp. 35-79.

- (1987), «Goal, event, and understanding in Ifaluk emotion theory», en Holland, Dorothy, y QuinN, Noami (eds.), Cultural models in language and thought, Cambridge, Cambridge University Press, pp. 35-79.

- (1988), Unnatural emotions: everyday sentiments on a Micronesian atoll and 
their challenge to Western theory, Chicago, University of Chicago Press.

- (1995), Need, nurtuance, and the emotions on a Pacific atoll, en MARKs, Joel, y Ames, Roger T. (eds.), pp. 253-252.

Marks, Joel, y AmES, Roger T. (eds.) (1995), Emotions in Asian thoughts: a dialogue in comparative philosophy, Albany, New York, State University of New York Press.

Markus, Hazel Rose, y Kitayama, Shinobu (1994), «The cultural construction of self and emotions: implications for social behavior», en Kitayama, Shinobu, y Markus, Hazel Rose (eds.), Emotion and culture: empirical studies of mutual influences, Washington DC, American Psychological Association.

Owen, Stephen (1985), Traditional Chinese poetry and poetics: omen of the world, Madison, Wisconsin, University of Wisconsin Press.

Plutchik, Robert (1994), The psychology and biology of emotion, New York, Harper Collins.

Qian, Zhongshu (1998), Guan Zhui Pian (Limited views: essays on ideas and letters) (selección y traducción de Ronald Egan), Massachusetts, Harvard University Asia Center.

Rosaldo, Michelle Z. (1980), Knowledge and passion: Ilongot notions of self and second life, Cambridge, Cambridge University Press.

Russell, James A. (1991), «Culture and the categorization of emotion», Psychological Bulletin, núm. 110, pp. 426-50.

Russell, James A., y Yıк, Michelle (1996), «Emotion among the Chinese», en Harris Bond, Michael (ed.), Handbook of Chinese Psychology, Hong Kong, Oxford University Press, pp. 166-88.

Santangelo, Paolo (2000), «Emotions in history and literature: An interdisciplinary research on emotions and states of minds in Ming-Qing period», Mingqing Yanjiu, pp. 237-308.
Scherer, Klaus R. (1994), Towards a concept of «Modal Emotions», en EKMAN, Paul, y Davidson, Richard J. (eds.), pp. 32-44.

Schwartz, T.; White, Geoffrey M., y Lutz, Catherine (eds.) (1992), New directions in psychological anthropology, Cambridge, Cambridge University Press.

Shaver, P.; Schwartz, J.; Kirson, D., y O'Connor, C. (1987), «Emotion knowledge: further exploration of a prototype approach», Journal of Personality and Social Psychology, núm. 52, pp. 1061-1086.

Shaver, P.; Wu, S., y Schwartz, J. (1992), «Cross-cultural similarities and differences in emotion and its representation», en Clark, Margaret S. (ed.), Emotion. Review of Personality and Social Psychology, núm. 13, pp. 175-212.

SHWEDER, Richard A. (1991), Thinking through cultures, Cambridge, Cambridge University Press.

- (en prensa), «The cultural psychology of the emotions: ancient and new», en Lewis, Michael, y Haviland, Jeanette (eds.), The Handbook of emotions, 2. ed., New York, Guiford Publications. Shweder, Richard, y LeVine R. A. (eds.) (1984), Culture theory: essays on mind, self, and emotion, Cambridge, Cambridge University Press.

Solomon, Robert C. (1995), The cross-cultural comparison of emotion, en MARKS, Joel, y Ames, Roger T. (eds.), pp. 253-308.

Travis, Catherine (en prensa), La Metalengua Semántica Natural: the Natural Semantic Metalanguage of Spanish, en Goddard y Wierzbicka (eds.).

VAN BRAKEL, Jaap (1993), «Emotions: a cross-cultural perspective on forms of life», en Wentworth, W. M., y Ryan, J. (eds.), Social perspectives on emotion, vol. II, Greenwich, JAI, pp. 179-237. Watson, James L., y Rawski, Evelyn S. (1988), Death Ritual in Late Imperial and Modern China, Berkeley, University of California Press. 
White, Geoffrey M., y KirkPatrick, John (1985), Person, self, and experience: exploring Pacific ethnopsychologies, Berkeley, California, University of California Press.

Wierzbicka, Anna (1972), Semantic primitives (Linguistische Forschungen, 22), Frankfurt am Main, Athenäum.

- (1980), Lingua Mentalis: the semantics of natural language, Sydney, Academic.

- (1986), «Human emotions: universal or culture-specific?», American Anthropologist, núm. 82, pp. 759-81.

- (1992), «Defining emotion concepts», Cognitive Science, núm. 16, pp. 539-81.

- (1995), «Everyday conceptions of emotion: a semantic perspective», en RusSell, James A.; Fernández-Dols, José Miguel; Manstead, Antony, y WellenKAMP, J. C. (eds.), Everyday conceptions of emotion: an introduction to the psychology, anthropology, and linguistics of emotion, Dordrecht y Boston, Kluwer Academic, pp. 17-47.

- (1996), Semantics: primes and universals, New York, Oxford University Press.

- (1999), Emotion across languages and cultures: diversity and universals, Cambridge University Press.

XIN, Shibiao (1993), «"Shangchun Beiqiu” jiqi wenhua tanyuan» [ «Shangchun Beiqiu» and its cultural origin], en CHEN, Jianming, y TAN, Zhiming (eds.), Yuyan yu wenhua duoxueke yanjiu (Cross-discipline studies of language and culture), Beijing, Beijing Yuyan Chubanshe.
YE, Zhengdao (1998), «Ming ruo qin xuan» (Life on a string)-Chinese ethno theory of life, manuscrito no publicado, Australian National University.

- (2000a), The language of emotions in Chinese: a study based on Hong Lou Meng, M. A. tesis, Australian National University.

- (en prensa) (a), An inquiry into «sadness» in Chinese, en Harkins, Jean, y WierzBicka, Anna (eds.).

- (2000b), «Cultural Scripts», Examples from Chinese emotional expression, paper presented at Workshop on Ethnopragmatics, Australian Linguistic Institute, University of Melbourne.

- (en prensa) (b), «Different modes of describing emotions in Chinese: bodily change, sensations, and bodily images», Pragmatics and Cognition.

- (en preparación) (a), Folk model of Chinese facial expressions: a linguistic perspective.

- (en preparación) (b), Emotionality and Chinese facial expressions.

Zhang, Fa (1989), Zhongguo wenhua yu beiju yishi (Chinese culture and the sense of tragedy), Beijing, Zhongguo Renmin Daxue Chubanshe.

La autora tiene una deuda especial con la profesora Anna Wierzbicka por su continuo estímulo y apoyo. Su influencia intelectual es visible en este trabajo.

\section{NOTAS}

${ }^{1} N$. del T. El análisis comparativo y contrastivo que se lleva a cabo en este trabajo es entre el chino y el inglés, por lo cual he decidido, según las circunstancias, traducir los términos de emoción al español o dejarlos en inglés. La equivalencia que establezco entre inglés y español es la siguiente: sadness/tristeza; sorrow/pena; grief/dolor; mourning/duelo, aunque en algunos casos utilizo otros términos que expresan mejor los matices de significado al traducir los ejemplos literarios.
2 Para un tratamiento detallado de la combinatoria de estos primitivos véase Travis (en prensa). Para los exponentes chinos de los primitivos semánticos y su gramática véase Chappell (1994, en prensa). La búsqueda empírica de conceptos humanos universales se ha expandido desde los 14 originales (Wierzbicka, 1972) hasta 60 en el presente (Goddard y Wierzbicka, 1994). Actualmente, el proyecto está acelerando su ritmo en la búsqueda de una gramática universal —la 
conducta combinatoria universal de estos conceptos humanos universales (Goddard y Wierzbicka, en prensa)—. Este trabajo adopta los últimos desarrollos y descubrimientos en el proyecto del MSN que apunta a definir los significados en un grado máximo de universalidad.

${ }^{3}$ Algunos de los ejemplos de textos que son usados en este trabajo también son tomados de Hongloumeng (véase Ye, 2000a, para un estudio detallado del sistema codificador de las emociones y sus significados en la lengua china basado en Hongloumeng). Algunas de las muchas consideraciones sobre la popularidad e importancia de esta novela es su inclusión como texto obligatorio para los estudiantes de high school en China (véase Cao y Gao, 2000). Gran parte de la poesía citada en este trabajo ha sido llevada a la música popular.

${ }^{4}$ Véase Santangelo (2000) para una discusión sobre la utilización de textos literarios como clave para reconstruir la vida emocional y mental de la gente en la historia.

${ }^{5}$ La romanización utilizada a lo largo de este trabajo es la Pinyin. Para facilitar la notación, los tonos no han sido marcados. La mayoría de los ejemplos lingüísticos siguen una notación tripartita - la primera línea en la romanización del pinyin, la segunda línea como notación morfema por morfema, seguida por la traducción literal-. Donde los textos son muy largos sólo se presentan las traducciones al inglés. Donde las fuentes son inespecíficas, la traducción es literal. Las abreviaciones utilizadas para la notación morfema por morfema son las siguientes: $\mathrm{CL}=$ clasificador; DUR $=$ durativo; PART $=$ partícula $;$ PFV $=$ aspecto perfectivo; PROG $=$ progresivo.

${ }^{6}$ Una explicación es una definición formulada por medio de una configuración de primitivos semánticos. Toma la forma de una serie de componentes, que está en concordancia con la visión general de los psicólogos de que las emociones son «fenómenos multicomponenciales» (Sherer, 1994; Fridja, Sato y Wiers, 1995). El marco semántico para explicar los conceptos de emoción adoptado aquí ha sido desarrollado por Wierzbicka (e. g., 1972, 1986, 1992, 1999). Pueden encontrarse explicaciones más detalladas de los fundamentos del marco del MSN para las palabras de emoción, y cómo refleja y coincide con los últimos desarrollos de los descubrimientos en los estudios de emociones de psicólogos y antropólogos, en Harkin (1990), Harkins y Wierzbicka (1997, en prensa), y Ye [2000a, en prensa a) y b)].

7 Es la elucidación del significado de bei la que ofrece una explicación con respecto a por qué bei, no $a i$, es propuesta como una emoción básica en los textos taoistas. Esto no debe interpretarse de manera inversa Tal vez sea porque no se ha hecho ningún intento previo para revelar el significado de bei que los estudiosos nunca han podido ver la «lógica» que está detrás de esta postulación de las «emociones chinas básicas».

${ }^{8}$ Springs and automns follow on in succession, with the brooding gloom of drak Yin and the easyfull brightness of Yang. And as the bright countenances of physical things are impelled in their cycles, so the affective capability of mind (hsin) too is shaken... And when autumn's skies are high and the animating air takes on a chill clarity, our thoughts, sunken in the darkness of Yin, touch on far things. The year has its physical things, and these things have their countenance; by these things our emotions are shifted, and from emotions languages comes. The fall of a solitary leaf finds its place in our understanding [and we know that automn is coming] (tr. Owen, 1985; el énfasis es mío).

${ }^{9} \mathrm{Si}$ («pensamientos/reflexiones/amorosos») juega un papel esencial en las emociones chinas. Es considerada una de las «emociones chinas básicas» en los textos tradicionales y forma su propia categoría distintiva. Liang (1996: 29) señala que, en comparación con el modelo de emociones multidimensional de Plutchik (1994), la clasificación china de las emociones básicas tiene una categoría si enfática que no puede ser contenida dentro del modelo de Plutchik. 